\title{
La educación como apoyo en el Fortalecimiento de la cultura Tributaria. Caso: NAF-UCACUE
}

\section{Education as support for the strengthening of tax culture. Case: NAF- UCACUE}

Patricio Mendieta

Universidad Católica de Cuenca, Ecuador

Autor por correspondencia: mendietaandrade@gmail.com

Fecha de recepción: 18 de septiembre de 2018 - Fecha de aceptación: 01 de diciembre de 2018

Resumen: Cumpliendo con su apostolado de servicio al pueblo, la Universidad Católica de Cuenca pone en marcha en el año 2016 el Proyecto Núcleo de Apoyo Contable y Fiscal, con el objetivo de constituirse en un actor positivo en la difusión de los conocimientos inherentes a los tributos en el país, y apoyando al fortalecimiento de la cultura tributaria de la ciudad de Cuenca. Con éste antecedente, el Proyecto ha organizado 17 conferencias y charlas hasta agosto del 2018, impartidas por especialistas y estudiantes de la Universidad, a fin de socializar el conocimiento inherente al cumplimiento tributario y su importancia, en los contribuyentes del cantón. Es así que, con el objetivo de cuantificar el efecto de las anotadas conferencias en la cultura tributaria de los asistentes, se efectuó las correspondientes encuestas, mismas que sirvieron de base para el análisis estadístico, donde se reconoce la relación entre las variables estudiadas, identificando una mejora en la percepción sobre los temas planteados luego de recibir la capacitación, por lo que queda en evidencia el rol importante que cumple la educación y concretamente la Universidad en el fortalecimiento de la cultura tributaria en nuestro país.

Palabras clave: cultura tributaria; educación

Abstract: Fulfilling its apostolate of service to the people, the Catholic University of Cuenca launched in 2016 the Core Project of Accounting and Fiscal Support, with the aim of becoming a positive actor in the dissemination of the knowledge inherent to the taxes in the country, and supporting the strengthening of the tax culture of the city of Cuenca. With this background, the Project has organized 17 conferences and talks until August 2018, given by specialists and students of the University, in order to socialize the knowledge inherent to tax compliance and its importance, in the taxpayers of the canton. Thus, in order to quantify the effect of the annotated conferences in the tax culture of the attendees, the corresponding surveys were carried out, which served as the basis for the statistical analysis, where the relationship between the variables studied is recognized, identifying an improvement in the perception of the issues raised after receiving the training, so it is evident the important role that education plays and specifically the University in strengthening the tax culture in our country.

Key words: tax culture; education 


\section{Introducción}

La Universidad Católica de Cuenca, consiente de su rol dentro de la sociedad cuencana y en el marco del Acuerdo de Cooperación Interinstucional entre la Universidad Católica de Cuenca (UCACUE) y el Servicio de Renta Internas (SRI), implementó el Proyecto de Vinculación con la Colectividad denominado Núcleo de Apoyo Contable y Fiscal (NAF), con el fin de constituirse en un centro gratuito de difusión del conocimiento contable y tributario, instruyendo a sus beneficiarios sobre el sentido social de los impuestos y el cumplimiento de los deberes formales como contribuyentes (UCACUE, 2016).

De esta manera, la UCACUE en el año 2016 instituye este espacio dentro de su organización, en el cual autoridades universitarias, profesores y estudiantes, se constituyen en miembros activos de la sociedad, capaces de generar un aporte positivo al fortalecimiento de la cultura tributaria en el cantón Cuenca, mediante la difusión de los conocimientos inherentes al cumplimiento tributario.

Como antecedente a lo expuesto, cabe anotar que el NAF de la UCACUE entre otras actividades que viene llevando a cabo a fin de lograr la consecución de sus objetivos y consiente de la importancia que tiene la educación como base de un cumplimiento tributario voluntario y efectivo, se propuso organizar un conjunto de conferencias y charlas informativas sobre temas relacionados a los impuestos en nuestro país; las cuales, fueron dictadas en la mayoría de los casos por expertos del SRI y en otros casos por estudiantes de la UCACUE; las cuales, fueron dirigidas a diferentes sectores de la sociedad, tales como adultos mayores, familiares y personas con discapacidades, alumnos de nuestra Instituciones, profesionales del área contable y público en general.

Por lo expuesto, desarrollamos el presente trabajo investigativo con el fin de cuantificar y exponer los resultados obtenidos por del NAF de la UCACUE durante el período indicado. Es así que proponemos como hipótesis de investigación que las conferencias organizadas por el NAF de la UCACUE durante el período 2106 a 2108 tienen un efecto positivo en el fortalecimiento de la cultura tributaria en los asistentes. Si bien es complicado establecer relaciones claras en este tipo de trabajo de carácter etnográfico, buscamos identificar por medio del registro de las percepciones de los asistentes, relaciones estadísticas que den pistas de una mejora en los conocimientos de los asistentes y su percepción de Sistema Tributario Ecuatoriano.

El presente documento expone, luego de ésta introducción, los resultados de la revisión del marco teórico referente a la problemática planteada, posterior a lo cual se especifica la metodología que se utilizó en el cumplimiento de los objetivos trazados en ésta investigación. En un tercer apartado se presentan los resultaos de la investigación, los mismos que son la base para que en la última sección se presenten las correspondientes conclusiones del caso.

\section{Materiales y Métodos}

\section{Marco Teórico}


El hacer el análisis de la estructura de la Proforma Presupuestaria del Ecuador para el ejercicio fiscal 2017, se evidencia la importancia que tiene la recaudación tributaria en las finanzas nacionales, pues ésta representan aproximadamente el $40 \%$ del total de los ingresos proyectados para dicho período (Ministerio de Economía y Finanzas, 2017), siendo evidente que el gasto que realiza el Gobierno en educación, salud, defensa nacional y otros, se sustenta en un elevado porcentaje, en los tributos que recauda.

Al revisar las cifras presentadas por el SRI, órgano estatal gestor de los tributos en nuestro país, se evidencia que en el año 2017 hubo un recaudo neto, es decir sumado las contribuciones solidarias y descontadas devoluciones, notas de crédito y compensaciones, de un valor de 12.725 millones de dólares; que, comparado con la meta propuesta por el ente, se reconoce un cumplimiento de la misma de un 92\% (SRI, 2018).

En atención a los datos presentados, se reconoce la importancia que reviste la función del SRI en el andamiaje económico nacional; por lo cual, el ente ha venido implementando cambios en su modelo de gestión, donde es evidente la importancia que se ha dado al fortalecimiento de la cultura tributaria, como eje fundamental en el que sustente el crecimiento de los recaudos tributarios; siendo una prueba de lo anotado el cambio del eslogan del órgano de "para cumplir y hacer cumplir" al ya reconocido "SRI le hace bien al país" (Quintanilla, 2012).

Dados los antecedentes anotados, cabe definir el término ya anotado de cultura tributaria; el cual según Armas y Colmenares (2009) se entiende como "el conjunto de valores, conocimientos y actitudes compartidos por los miembros de una sociedad respecto a la tributación y la observancia de las leyes que la rigen"(p.148); siendo que, el cumplimiento tributario de forma consiente y voluntario se constituyen en el factor fundamental para la consecución de las metas de recaudación fiscal planteadas desde el Ejecutivo (Sarmiento, 2014).

Por lo expuesto, se puede afirmar que la cultura tributaria constituye la base en la que se sustenta, en el largo plazo, un sistema tributario eficiente; siendo que, la actitud del contribuyente es fundamental como garantía del cumplimiento de los tributos. Es así, que a fin de logra un fortalecimiento de la cultura tributaria será importante que los miembros de la sociedad adquieran los conocimientos y reconozcan la importancia de sus responsabilidades tributarias, mediante un sistema de información oportuno y adecuado (Arriaga, Reyes, Olives, \& Solórzano, 2017), logrando una armonía entre lo cultural y lo ético, donde el contribuyente cumpla con sus impuestos por un deber cívico y no solo por cumplimiento a la Ley (Soto, 2016).

En línea con lo expuesto, Marx Carrasco (2010), Director del SRI en el período 20072014, afirma que el Gobierno Nacional en el proyecto de la llamada Revolución Ciudadana fue el primero en reconocer al ser humano como sujeto y fin de la relación entre la sociedad y el Estado; siendo que, en el marco de tres conceptos básicos, tales como la ética reflejada en aquellos comportamientos moralmente aceptados, el pacto social y finalmente la cohesión social, vista ésta como la "concienciación individual y colectiva de los compromisos y responsabilidades dentro de la comunidad", se constituye la base para consolidar el concepto de ciudadanía fiscal; la cual es definida por el autor como " la actitud consiente y voluntaria de mujeres y hombres en el cumplimiento de sus obligaciones fiscales, como condición para el ejercicio pleno de sus derechos"(p. 16). 
Con el marco precedente y partiendo de la importancia de cuantificar las diferentes variables implícitas en la dinámica social, los autores Méndez, Morales y Aguilera (2005), consideran que la cultura tributaria se debería evaluar desde la perspectiva socio-cultural y no desde el punto de vista de las variaciones de los saldos de las cuentas nacionales. Es así que, en el trabajo de López (2012), al estudiar a los comerciante del Barrio el Beaterio de la ciudad de Quito, se evidencia que el 64\% de la muestra estudiada, rara vez o nunca considera que la tributación es parte fundamental para la economía del Estado; aunque el mayor porcentaje de la muestra reconoce la gravedad de las infracciones tributarias, la mayoría desconoce cuáles son sus obligaciones tributarias.

De la misma manera, Bedoya y Vásconez (2010) al realizan una evaluación de la moral tributaria pone en evidencia, en una muestra de 4160 personas tomadas en 11 ciudades del Ecuador, que el $35.43 \%$ de los encuestados considera que en ciertas ocasiones las personas o empresas pueden tener alguna justificación para no pagar sus impuestos y un $4.3 \%$ considera justificable el no pagar sus impuestos. Los autores establecen una fuerte relación entre los valores de los encuestados, la confianza de los contribuyentes en el manejo de los ingresos tributarios por parte del Gobierno, el conocimiento del sistema tributario y el pago de los impuestos.

En su trabajo, los autores Arriaga, Reyes, Olives y Solórzano (2017) en su estudio reconoce como causas de una deficiente cultura tributaria, para el caso de las personas que pagan el Impuesto a la Renta en la Provincia de Santa Elena, en su orden de importancia, el desinterés en el pago de los impuestos y el desconocimiento de los mismos; los cuales, inciden en el incumplimiento tributario. Por otro lado, en el mismo trabajo se evidenció que en el $45 \%$ de los casos estudiados no se re considera importante el pago de los impuestos y el beneficio que éstos aportan al desarrollo del país.

Por lo expuesto y coincidiendo con varios trabajos previos al presente (Briseño,2010; Fernández \& Calero, 2011; Mendoza, Palomino, Robles, \& Ramírez, 2016; Robles, \& Ramírez, 2016, López, 2012), se considera que la educación es uno de los principales factores que determinan el desarrollo sostenido y generalizados de una sociedad; más, en el plano del cumplimiento tributario, es fundamental un sistema educativo que apoye al fortalecimiento de la cultura tributaria, mediante la socialización de los conocimientos inherentes a los tributos y el reconocimiento de la importancia de los mismos en el desarrollo equitativo y justo de la economía nacional.

\section{Metodología}

El presente estudio se enmarca como una investigación de carácter cualitativo, en el que "la meta es el desarrollo de conceptos que ayuden a entender fenómenos sociales dando énfasis a los significados, experiencias y opiniones de todos los actores sociales" (Pérez \& Souza, 2013). Se puede anotar que los datos analizados provienen de las encuestas realizadas a los asistentes a las 17 conferencias y charlas informativas organizadas por el NAF de la UCACUE durante el período 2016 a 2018, donde se contabilizaron aproximadamente 300 asistentes en total, lográndose recabar 260 respuestas dentro de los concurrentes; siendo posible anotar, que se trabajó con una muestra que refiere un nivel de confianza superior al $97 \%$. 
A fin de realizar la evaluación del efecto de las capacitaciones en los aspectos inherentes en la cultura tributaria en los asistentes a las conferencias, se diseñó el cuestionario correspondiente en el que se buscó evidenciar su opinión. En el instrumento se optó por la utilización de la escala de Likert, siendo que las posibles respuestas fueron 1 totalmente en desacuerdo, 2 en desacuerdo, 3 ni de acuerdo ni en desacuerdo, 4 de acuerdo y 5 totalmente de acuerdo a diferentes afirmaciones que se plantearon.

Se utilizó como modelo de predicción el árbol de decisión, a fin de realizar un sondeo y exploración de los resultados obtenidos, creando un modelo de clasificación que, sustentado en un diagrama de flujo, logre clasificar los casos en grupos, pronosticando los valores de la variable dependiente, basados en los valores que asuma la o las variables independientes. Para tal efecto se utilizó el método CHAID (detector de interacción automática Chi-cuadrado), siendo definido este como "un rápido algoritmo de árbol estadístico, multidireccional que explora datos de forma rápida y eficaz, y crea segmentos y perfiles con respecto al resultado deseado; permitiendo, la detección automática de interacciones mediante Chi-cuadrado" (Berlanga, Rubio, \& Vilá, 2013, pág. 68).

El análisis de datos se realizó mediante el programa SPSS y Excel; siendo que para el análisis cualitativo se utilizaron los estadísticos descriptivos y tablas de contingencias; para el análisis inferencial se aplicó la prueba de Chi-cuadrado y otros estadísticos para realizar las correspondientes pruebas de hipótesis de asociatividad, tales como el coeficiente de correlación de Spearman.

\section{Resultados}

Luego de aplicado el instrumento de investigación, se obtuvo como respuesta al enunciado: "luego de asistir a la conferencia mejoraron sus conocimientos inherentes al cumplimiento tributario", que el 93.90\% de los encuestados respondió estar de acuerdo o totalmente de acuerdo con la misma; siendo que el 3.10\% responde que está en descuerdo o totalmente en desacuerdo con la afirmación. Al proponer el enunciado: "los conocimientos que adquirió en la capacitación serán de mucha utilidad en el futuro para lograr un cumplimiento eficaz de sus obligaciones tributarias". El 95.70\% anota que está de acuerdo o totalmente de acuerdo, mientras que el $1.90 \%$ está en desacuerdo o totalmente en desacuerdo con la afirmación planteada. Al realizar el análisis cualitativo de los resultados obtenidos, se puede afirmar que las capacitaciones realizadas por el NAF de la UCACUE mejoran los conocimientos referentes a las obligaciones tributarias y son considerados como útiles en el cumplimiento de las mismas en un la mayor parte de los asistentes; siendo que en una proporción pequeña no se logra el objetivo planteado en las capacitaciones dictadas.

En este punto se muestran los resultados al aplicar la técnica de segmentación CHAID, siendo que el modelo toma como variable dependiente el mejoramiento de los conocimientos inherentes al cumplimiento de los tributos y como variables independientes o predictoras las respuestas a los enunciados que se plantearon en el cuestionario y se detallan a continuación:

- El tema causó mucho interés en el asistente.

- La exposición cubrió las expectativas en el asistente. 
- El tema tratado quedó completamente explicado.

- Con la asistencia a la capacitación comprende completamente la temática.

Siendo que las respuestas posibles van del 1 al 5, según la escala de Likert descrita previamente, cabe anotar que 0 se asignó a los casos en los que no hubo respuesta al planteamiento. Es así que la Figura 1, se muestra el diagrama de árbol para el modelo del mejoramiento de los conocimientos inherentes al cumplimiento de los tributos.

1. Mejoraron su conocimientos inherentes al cumplimiento

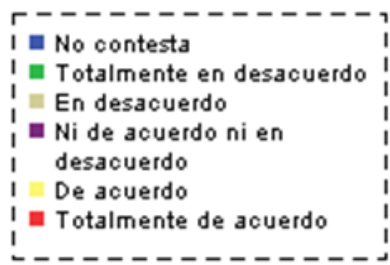

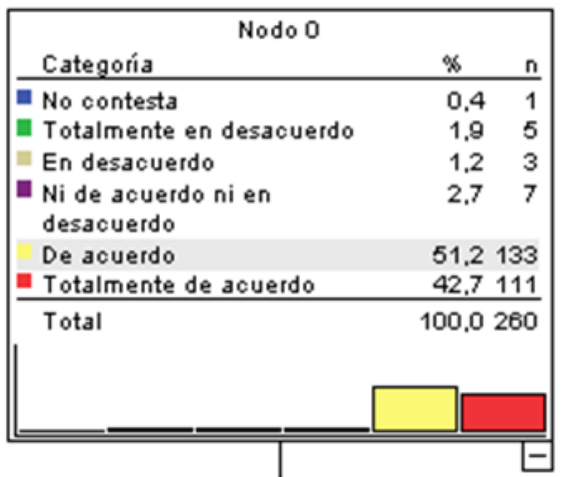

B La exposición cubrió las expectativas en el asistente.

Valor $P$ corregido $=0,000$, Chi-cuadrado $=38,989, d f=1$

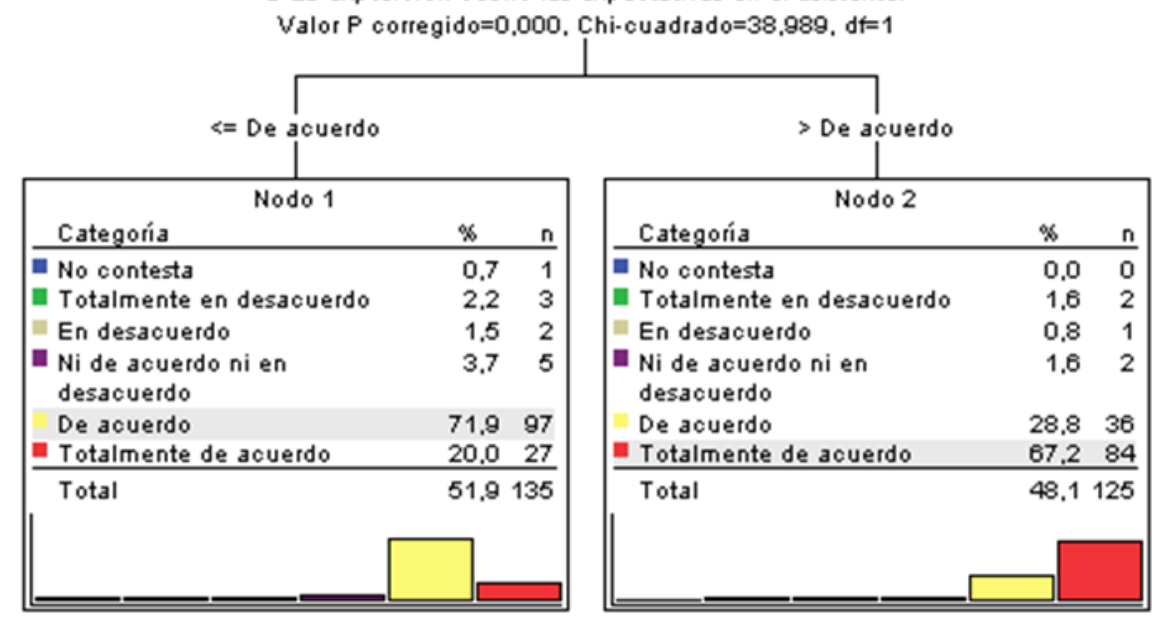

Figura 1. Diagrama de árbol para el modelo de mejoramiento del conocimiento inherente al cumplimiento de los tributos.

Nota. Fuente: elaboración propia en base a los resultados de la encuesta.

Al interpretar los outputs que se obtuvieron al general el árbol en el programa SPSS, se puede anotar que el modelo considera únicamente a la respuesta a la afirmación de que la exposición cubrió las expectativas de los asistentes como variable independiente incluida en el modelo; siendo evidente un Chi-cuadrado de 38.989 con un Pvalor de 0.000. Es así que la variable satisfacción de las expectativas de los asistentes a las capacitaciones es la mejor predictora del mejoramiento de los conocimientos inherentes al cumplimiento de las obligaciones tributarias, con dos categorías menor o igual a de acuerdo y totalmente de acuerdo.

Se anota que la probabilidad más alta de mejorar los conocimientos en materia tributaria (71.9\%) se da en los asistentes que están de acuerdo en que el tema tratado cubrió sus 
expectativas. Por otro lado, lo anotado es coherente con la probabilidad más baja de mejorar los conocimientos $(0.8 \%)$ obtenida; la cual se da entre los asistentes que están en desacuerdo con la afirmación: "la exposición cubrió las expectativas en el asistente". Posteriormente, al evaluar la bondad de funcionamiento del modelo, la tabla de clasificación muestra que el modelo clasifica de forma correcta un $69.6 \%$, aproximadamente, de las observaciones en general.

De la misma manera, a fin de realizar el examen del efecto de las conferencias en la cultura tributaria de los asistentes, se planteó el análisis a nivel descriptivo de la muestra, evaluando la existencia de algún tipo de relación entre el interés que causó el tema tratado y las respuestas de los encuestados a la afirmación: "luego de recibir la capacitación, considera que los conocimientos adquiridos serán de utilidad en el cumplimiento eficaz de sus obligaciones tributarias", planteada en el correspondiente cuestionario. Para tal efecto se construyó la tabla de contingencia pertinente, y se reconoce que el $73.3 \%$ de los encuestados, luego de recibir la capacitación, están totalmente de acuerdo en considerar interesante el tema tratado, están totalmente de acuerdo que los conocimientos que adquirió serán de utilidad para el cumplimiento de sus impuestos (ver Tabla 1).

Tabla 1. Tabla de contingencia: Los conocimientos que adquirió le serán de mucha utilidad en el futuro para poder cumplir de forma eficaz sus impuestos - El tema causo mucho interés en el asistente

El tema causo mucho interés en el asistente.
$\begin{array}{lllllll}\text { No } & \text { Totalment } & \text { En } & \text { Ni de } & \text { De } & \text { Totalment } & \text { Total } \\ \text { contest } & \text { e en } & \text { desacuerd } & \text { acuerdo ni } & \text { acuerd } & \text { e de } & \\ \text { a } & \text { desacuerd } & \text { o } & \text { en } & \text { o } & \text { acuerdo } & \\ & \text { o } & & \text { desacuerd } & & & \end{array}$

\begin{tabular}{|c|c|c|c|c|c|c|c|c|}
\hline \multirow{8}{*}{$\begin{array}{l}\text { Los } \\
\text { conocimiento } \\
\text { s que } \\
\text { adquirió le } \\
\text { serán de } \\
\text { mucha } \\
\text { utilidad en el } \\
\text { futuro para } \\
\text { poder } \\
\text { cumplir de } \\
\text { forma eficaz } \\
\text { sus } \\
\text { impuestos. }\end{array}$} & \multirow{3}{*}{$\begin{array}{l}\text { No } \\
\text { contesta }\end{array}$} & & & & \multicolumn{4}{|l|}{ o } \\
\hline & & 2 & 0 & 0 & 0 & 0 & 0 & 2 \\
\hline & & $100,0 \%$ & $0,0 \%$ & $0,0 \%$ & $0,0 \%$ & $0,0 \%$ & $0,0 \%$ & $\begin{array}{l}100,0 \\
\%\end{array}$ \\
\hline & Totalment & 0 & 3 & 0 & 0 & 0 & 1 & 4 \\
\hline & $\begin{array}{l}\text { e en } \\
\text { desacuerd } \\
\text { o }\end{array}$ & $0,0 \%$ & $75,0 \%$ & $0,0 \%$ & $0,0 \%$ & $0,0 \%$ & $25,0 \%$ & $\begin{array}{l}100,0 \\
\%\end{array}$ \\
\hline & En & 0 & 0 & 0 & 0 & 1 & 0 & 1 \\
\hline & $\begin{array}{l}\text { desacuerd } \\
0\end{array}$ & $0,0 \%$ & $0,0 \%$ & $0,0 \%$ & $0,0 \%$ & $\begin{array}{l}100,0 \\
\%\end{array}$ & $0,0 \%$ & $\begin{array}{l}100,0 \\
\%\end{array}$ \\
\hline & Ni de & 0 & 0 & 1 & 1 & 2 & 0 & 4 \\
\hline $\begin{array}{l}\text { Sus } \\
\text { impuestos. }\end{array}$ & $\begin{array}{l}\text { acuerdo ni } \\
\text { en } \\
\text { desacuerd } \\
\text { o }\end{array}$ & $0,0 \%$ & $0,0 \%$ & $25,0 \%$ & $25,0 \%$ & $50,0 \%$ & $0,0 \%$ & $\begin{array}{l}100,0 \\
\%\end{array}$ \\
\hline & De & 1 & 1 & 0 & 4 & 73 & 35 & 114 \\
\hline & acuerdo & $0,9 \%$ & $0,9 \%$ & $0,0 \%$ & $3,5 \%$ & $64,0 \%$ & $30,7 \%$ & $\begin{array}{l}100,0 \\
\%\end{array}$ \\
\hline & Totalment & 1 & 2 & 0 & 1 & 32 & 99 & 135 \\
\hline & $\begin{array}{l}\text { e de } \\
\text { acuerdo }\end{array}$ & $0,7 \%$ & $1,5 \%$ & $0,0 \%$ & $0,7 \%$ & $23,7 \%$ & $73,3 \%$ & $\begin{array}{l}100,0 \\
\%\end{array}$ \\
\hline Total & & 4 & 6 & 1 & 6 & 108 & 135 & 260 \\
\hline & & $1,5 \%$ & $2,3 \%$ & $0,4 \%$ & $2,3 \%$ & $41,5 \%$ & $51,9 \%$ & $\begin{array}{l}100,0 \\
\%\end{array}$ \\
\hline
\end{tabular}


Nota. Fuente: elaboración propia en base a los resultados de la encuesta.

Luego, al evaluar la existencia de una relación a nivel poblacional utilizamos la prueba de Chi-cuadrado de Pearson; para lo cual, se planteó la hipótesis nula, en la que se refiere la existencia de independencia entre las variables; siendo que, al analizar los resultados de la Tabla 2 , se evidencia un valor del estadístico de 349,062 con una significancia menor al 0.05 , por lo que rechazamos la hipótesis nula y afirmamos que existe evidencia estadística de que las variables son dependientes entre sí. Al evaluar la correlación de las variables estudiadas, se plantea la hipótesis nula de que no existe correlación entre las variables estudiadas, considerando un nivel de significancia de 5\%, siendo que utilizamos el coeficiente de correlación de Spearman, obtenemos un valor de Rho de 0,468 con un P-valor de 0,000 , por lo que rechazamos la hipótesis nula, reconociendo que existe una buena correlación positiva entre el interés que causaron los temas planteados en las conferencias y la apreciación de utilidad de los conocimientos adquiridos.

Tabla 2. Prueba Chi-cuadrado de Pearson

\begin{tabular}{llll}
\hline & Valor & gl & Sig. asintótica (bilateral) \\
\hline Chi-cuadrado de Pearson & 349,062 & 25 & 0,000 \\
Razón de verosimilitudes & 105,389 & 25 & 0,000 \\
Asociación lineal por lineal & 79,567 & 1 & 0,000 \\
N de casos válidos & 260 & & \\
\hline
\end{tabular}

Nota. Fuente: elaboración propia en base a los resultados de la encuesta.

Por otro lado, continuando con el análisis del efecto de las capacitaciones en la cultura tributaria de los asistentes a las conferencias organizadas por el NAF de la UCACUE, se comparó las percepciones de los asistentes, antes y después de las capacitaciones, a fin de cuantificar bajo los parámetros de la escala de Likert su respuesta a las afirmaciones planteadas. En la Figura 2 se presentan las medias de las respuestas obtenidas por los asistentes:

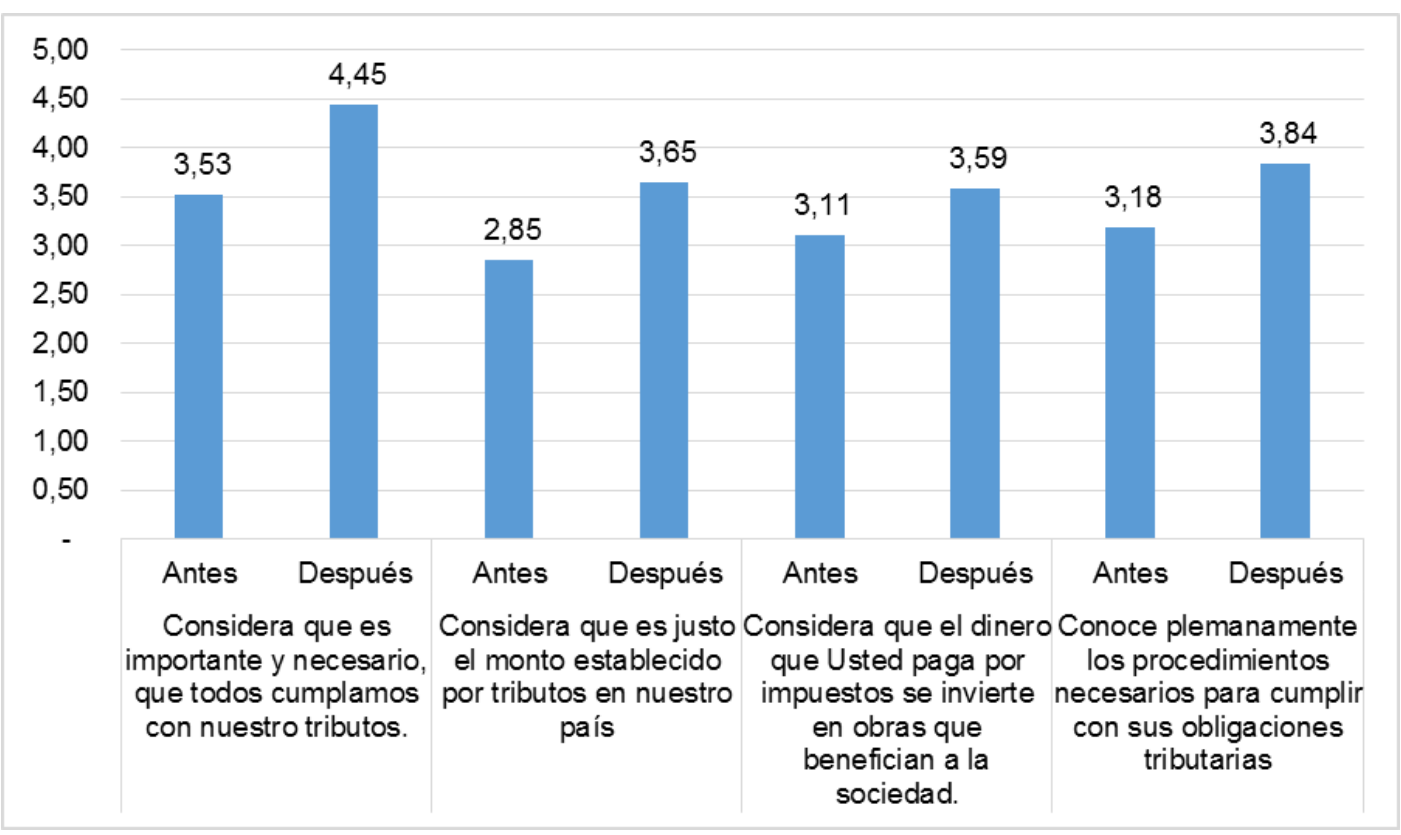

Figura 2. Media de los resultados de las percepciones de los asistentes a las conferencias. Nota. Fuente: elaboración propia en base a los resultados de la encuesta. 
Se puede observar en la Figura 2 que después de recibida la capacitación, los asistentes mejora su percepción sobre los aspectos planteados. Basados en estos datos y realizando el promedio de las cuatro respuestas por cada asistente, asignamos una calificación a la cultura tributaria (siendo menor que 3 malo, 3 regular y mayor que 3 bueno) a cada caso obteniendo los resultados que se presentan en la Tala 3:

Tabla 3. Tabla de contingencia: calificación de la cultura tributaria de los asistentes antes y después de recibir la conferencia.

\begin{tabular}{llllll}
\hline & \multicolumn{4}{l}{ Después de la capacitación } \\
\cline { 3 - 6 } & Malo & Regular & Bueno & Total \\
\hline \multirow{2}{*}{ Antes de la } & Malo & 16 & 25 & 44 & 85 \\
capacitación & Regular & 18 & 34 & 87 & 139 \\
& Bueno & 0 & 8 & 27 & 35 \\
\hline Total & & 34 & 67 & 158 & 259 \\
\hline
\end{tabular}

Nota. Fuente: elaboración propia en base a los resultados de la encuesta.

A fin de verificar estadísticamente la existencia de un cambio en alguna dirección en particular de los resultados obtenidos de la evaluación de la cultura tributaria por efecto de las capacitaciones impartidas, utilizamos la prueba de simetría de McNemar-Bowker, donde obtenemos un valor de 110,83 y un P-valor de 0,000 , por lo que se rechaza la hipótesis nula y se acepta la hipótesis de que la calificación cultura tributaria es distinta antes y después de asistir a las capacitaciones organizadas.

\section{Tabla 4. Resultados de la Prueba Chi-cuadro de McNemar-Bowker}

\begin{tabular}{llll}
\hline & Valor & gl & Sig. asintótica (bilateral) \\
\hline Prueba de McNemar-Bowker & 110,834 & 3 & 0,000 \\
N de casos válidos & 259 & & \\
\hline
\end{tabular}

Nota. Fuente: elaboración propia en base a los resultados de la encuesta.

\section{Conclusión}

En el presente trabajo de investigación se puso en evidencia la importancia del Sistema Tributario Ecuatoriano en las finanzas nacionales, siendo destacada la función que desempeña el SRI como organismo gestor de los impuestos en nuestro país.

De la misma manera es importante reconocer a la cultura tributaria, como base fundamental en el cumplimiento voluntario de los impuestos; siendo evidente la relación positiva entre una buena percepción del sistema tributario; la cual sumada a un conocimiento pleno de los aspectos que enmarcan los tributos, hace que ésta cultura se fortalezca y se constituya en garantía de una recaudación sostenida en el largo plazo.

Luego de aplicadas la encuestas y el análisis estadístico correspondiente se puede concluir que las conferencias organizadas por el NAF de la UCACUE durante el período 2106 a 
2108 si tienen un efecto positivo en el fortalecimiento de la cultura tributaria en los asistentes. Es así, que se reconoce la mejora en la percepción de la de la importancia de los tributos, la justicia en los montos establecidos por concepto de impuestos y el fin que se da a los valores recaudados, por parte de los encuestados.

Con los resultados expuestos, se reconoce el papel que tienen las universidades, como entes activos en la difusión del conocimiento inherente al cumplimiento tributario; por lo cual, se destaca la labor que realiza el NAF de la UCACUE en su objetivo de ser un apoyo al fortalecimiento de la cultura tributaria en el cantón Cuenca, siendo ciertos, lo resultados obtenidos con la organización de las conferencias y charlas durante el período de estudio.

Consideramos importante anotar que el presente trabajo se limita a un estudio de las percepciones de los asistentes bajo un enfoque investigativo de carácter netamente cualitativo; por lo cual, sería un gran aporte en el tratamiento de la temática, analizar el efecto que se produce con el fortalecimiento de la cultura tributaria desde el punto de vista de la variación de las recaudaciones, es decir bajo un enfoque cuantitativo.

\section{Bibliografía}

Armas, M., \& Colmenares, M. (2009). Educación para el desarrollo de la cultura tributaria. REDHECS, 6(4), 141-160. Obtenido de https://dialnet.unirioja.es/descarga/articulo/2937210.pdf

Arriaga, G., Reyes, M., Olives, J., \& Solórzano, V. (2017). Análisis de la cultura tributaria: Impuesto a la renta par personas naturales no obligadas a llevar contabilidad, Provincia de Santa Elena. Ciencias Pedagógicas e Innovación UPSE, 5(3), 118-127. Doi:DOI http://dx.doi.org/10.26423/rcpi.v5i3.192

Bedoya, A., \& Vascones, B. (2010). Entendiendo la moral tributaria en Ecuador. Revista Fiscalidad (5), 91-132. Obtenido de https://cef.sri.gob.ec/pluginfile.php/16836/mod_page/content/64/5_3.pdf

Berlanga, V., Rubio, M., \& Vilá, R. (2013). Cómo aplicar árboles de decisión en SPSS. Revista d'Innovació i Recerca en Educación, 6(1), 65-79. Obtenido de http://diposit.ub.edu/dspace/bitstream/2445/43762/1/618361.pdf

Carrasco, M. (2010). La ciudadanía fiscal: algunas reflexiones. Revista Fiscalidad (5), 11-66. Obtenido de https://cef.sri.gob.ec/pluginfile.php/16836/mod_page/content/64/5_2.pdf

Fernández, M., \& Calero, J. (2011). Los efectos no monetarios de la educación, Análisis a partir del consumo de los hogares. Revista de Educación, 419-442. Obtenido de http://www.revistaeducacion.mec.es/re355/re355_18.pdf

López, C. (2012). Efecto de la educación sobre el delito: evidencia para Argentina. (Tesis de maestría, Universidad Nacional de La Plata). Obtenido de 
http://sedici.unlp.edu.ar/bitstream/handle/10915/24608/Documento_completo__.pdf?sequ ence $=1$

López, F. (2012). Incidencia de los conocimientos sobre tributación y el cumplimiento de las obligaciones tributarias entre el pequeño comerciante del Barrio el Beaterio en el Sector de Guajaló. (Trabajo de grado). Universal Central del Ecuador, Quito, Ecuador.

Mendoza, F., Palomino, R., Robles, J., \& Ramírez, S. (2016). Correlación entre cultura tributaria y educación tributaria universitaria: Caso Universidad Estatal de Sonora. Revista Global de Negocios, 4(1), 61-76. Obtenido de ftp://ftp.repec.org/opt/ReDIF/RePEc/ibf/rgnego/rgn-v4n1-2016/RGN-V4N1-2016-5.pdf

Ministerio de Economía y Finanzas. (2017). Cifras del Presupuesto General del Estado 2018. Obtenido de https://www.finanzas.gob.ec/wpcontent/uploads/downloads/2017/11/Proforma_2018_para_Asamblea.pdf

Morales, N., Méndez, M., \& Aguilera, O. (2005). Cultura tributaria y contribuyente: datos y aspectos metodológicos. Fermentum. Revista Venezolana de Sociología y Antropología, 15(44), 332-352. Obtenido de http://www.redalyc.org/articulo.oa?id=70504404

Pérez, P., \& Souza, A. (2013). Como quantificar os aspectos cualitativos nas escalas de classificação de resultados NOC para etiquetas psicossocioculturais. Revista da Escola de Enfermagem da USP, 47(3), 728-735. Doi: DOI: 10.1590/S0080-623420130000300029

Quintanilla, J. (2012). La universidad en la cultura tributaria. RETOS. Revista de Ciencias de la Administración y Economía, 2(3), 105-114. Obtenido de http://www.redalyc.org/pdf/5045/504550953007.pdf

Sarmiento, B. (2014). La cultura tributaria en el Ecuador. Observatorio de la economía Latinoamericana (201). Obtenido de http://www.eumed.net/cursecon/ecolat/ec/2014/cultura-tributaria.html

Soto, M. (2016). La cultura tributaria: una contribución para la construcción colectiva del desarrollo sustentable. Novum Scientiarum, 2(1), 46-56. Obtenido de http://www.ecoambienteydesarrollo.org/revista/ojs/index.php/novum/article/view/62/cult uratributaria

SRI. (2018). Estadísticas generales de recaudación. Obtenido de http://www.sri.gob.ec/web/guest/estadisticas-generales-derecaudacion?p_auth=KeAzH2ym\&p_p_id=busquedaEstadisticas_WAR_BibliotecaPortle t_INSTANCE_EVo6\&p_p_lifecycle $=1 \&$ p_p_state=normal\&p_p_mode=view\&p_p_col_ id=column-1\&p_p_col_count=2\&_busquedaEstadisticas_

UCACUE. (2016). Acuerdo de cooperación interinstitucional entre la UCACUE y el SRI. [Acta convenio]. Archivo UACUE. 